\title{
Digitalisierung gestalten, nicht verwalten
}

\author{
M. Duzdar
}

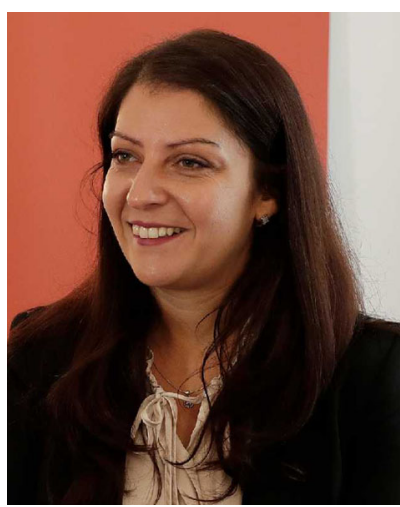

Mag. Muna Duzdar, Staatssekretärin im Bundeskanzleramt
3,7 Millionen Menschen in Österreich sind täglich auf Facebook aktiv. 18 Prozent haben bereits einmal als Crowdworker oder als Clickworker gearbeitet. Etwa 800.000 Menschen nutzen die Handysignatur, um Behördenwege über das Internet abzuwickeln. Einige wenige Beispiele, die deutlich machen, wie sehr die Welt mittlerweile von der Digitalisierung durchdrungen ist. Es ist das gesamte Leben. Egal, ob Beruf oder Freizeit, Ausbildung oder Alltag. Vom Kleinkind bis zum Pensionisten kom-

men alle Menschen täglich mit der Digitalisierung in Kontakt. Die Buchung des Urlaubs im Internet, der digitale Arbeitsplatz, die smart vernetzte Wohnung, der Kontakt mit Freunden und Bekannten in den sozialen Netzwerken. Ein Leben ohne Digitalisierung ist nicht mehr vorstellbar.

Doch was bedeutet das für uns, was bedeutet es für die Politik? Welcher Auftrag und welche Aufgaben sind damit verbunden? Digitalisierung bietet enorme Chancen und Möglichkeiten, aber auch Gefahren. Eine der Gefahren ist die Teilung der Bevölkerung in Menschen, die die Digitalisierung zu nutzen wissen und solchen, die nicht zu den Verständigen in diesem Prozess gehören. Wer heute nicht an der digitalen Welt teilnimmt, hat Nachteile zu befürchten. Bei der Suche nach einem Job, bei der Ausbildung, im täglichen Leben. Daher sehe ich es als Hauptaufgabe des Staates, die digitale Kluft zu schließen. Wir wissen, dass heute noch immer rund 13 Prozent der Menschen in Österreich das Internet noch nie genutzt haben. Und - auch wenn man es vermuten würde - das sind nicht ausschließlich Menschen höheren Alters. Die Nutzung des Internets ist auch eine Frage der sozialen Herkunft und auch des Geschlechts. Um in der heutigen Welt bestehen zu können, muss man aber nicht nur über den Zugang zur Digitalisierung verfügen, viel wichtiger ist es, den richtigen Umgang damit zu erlernen. Es ist schön, wenn die Durchdringung mit Smartphones in Österreich beinahe die höchste in Europa ist. Doch das reicht nicht aus. Medienkompetenz und Datenkompetenz sind mindestens ebenso essentiell. Wie kann ich richtige von falschen Meldungen unterscheiden? Wie kann ich mich wehren, wenn ich im Internet angegriffen werde? Hier gilt es konkrete Maßnahmen zu setzen.

\section{Digital Roadmap - die digitale Gesamtstrategie der Bundesregierung}

Dies sind zwei der Themen, die in der so genannten Digital Roadmap behandelt werden und die vielleicht nicht auf den ersten Blick mit dem Thema digitale Transformation verbunden werden. Die Digital
Roadmap ist die digitale Gesamtstrategie der Bundesregierung, die Anfang des Jahres präsentiert wurde. Unter insgesamt zwölf Leitprinzipien sind darin 150 konkrete Maßnahmen gebündelt, die Österreich in den kommenden Jahren zum digitalen Musterland machen sollen. Ziel der Roadmap war es, die vielen bestehenden Einzelinitiativen, die in den vergangenen Jahren in den verschiedenen Politikbereichen gestartet wurde, zu bündeln und mit einem Dach und einer Vision zu versehen. Denn Digitalisierung ist eine Querschnittsmaterie und muss auch dementsprechend behandelt werden. Eine weitere wesentliche Aufgabe dabei war es, weiße Flecken auf der Digitallandkarte zu schließen und Strategien in Bereichen zu entwickeln, mit denen wir uns bis jetzt noch nicht auseinander gesetzt haben. Jedes Jahr sollen diese Maßnahmen evaluiert und überprüft werden. Einerseits, um zu erkennen, ob die Maßnahmen auch umgesetzt werden, aber auch, um die Richtung der Maßnahmen zu überprüfen. Sind wir noch auf dem richtigen Weg? Wohin entwickeln sich andere Länder? In welche Richtung entwickelt sich die Digitalisierung? Wo muss man nachjustieren? Und in welchen Bereichen gibt es neue Entwicklungen, mit denen wir uns beschäftigen wollen oder müssen?

\section{Lernen von den anderen}

Von anderen zu lernen, ist wohl die entscheidende Grundlage, wenn man es sich zur Aufgabe gemacht hat, Österreich in der Digitalisierung nach vorne zu bringen. Dies war einer der Gründe, warum ich Anfang des Jahres Israel besuchte. Israel gilt als Hotspot der Digitalisierungsszene. Und das nicht umsonst. In dem kleinen Land, das etwa dieselbe Einwohnerzahl hat wie Österreich, entstehen pro Jahr etwa 5000 neue Start-ups. Das sind im Schnitt beinahe eineinhalb Start-ups pro Tag. Den Grund, warum es in Israel eine so hohe Dichte an neuen Start-ups gibt, sehe ich auch in gewisser Weise kulturell bedingt. In Israel ist die so genannte Kultur des Scheiterns deutlich stärker ausgeprägt als bei uns. Man darf scheitern, soll es sogar, denn aus Fehlern lernt man. So habe ich in Tel Aviv Start-upGründer getroffen, die mir erzählt haben, dass sie trotz eines tollen Produkts Schwierigkeiten hatten, Investoren zu finden, weil sie immer wieder folgenden Satz hörten: „Das ist euer erstes Start-up? Tolle Idee, aber wir warten lieber noch, bis ihr ein- oder zweimal gescheitert seid und dadurch Erfahrung gesammelt habt und etwas gelernt habt." Natürlich ist Scheitern keine Grundvoraussetzung für den Erfolg eines jungen Unternehmers, aber ein etwas entspannterer Umgang damit wäre tatsächlich manchmal von Vorteil. Solch ein Kulturwandel braucht Zeit und muss gestaltet werden.

Aber ich habe auch die Schattenseiten der Digitalisierung in Israel gesehen. Denn in Israel profitiert nur ein Bruchteil der Bevölkerung von der Digitalisierung. Die Vorteile kommen nur einigen wenigen tatsächlich zu Gute.

Und darum haben wir unsere digitale Roadmap auch unter zwei zentrale Motti gestellt. Einerseits sollen möglichst viele Menschen

Duzdar, Muna, Staatssekretärin für Diversität, Öffentlichen Dienst und Digitalisierung, Bundeskanzleramt, Ballhausplatz 1, 1010 Wien, Österreich

(E-Mail: muna.duzdar@bka.gv.at) 
von der Digitalisierung profitieren, und gleichzeitig gilt es, die Veränderungen zu gestalten und nicht nur zu verwalten. Aktive Maßnahmen sind der Schlüssel zu einer positiven Rezeption und damit auch zu einem positiven Wandel.

\section{Wandel von Anfang an gestalten}

Und dieser positive Wandel muss so früh wie möglich passieren und sich danach durch das gesamte Leben ziehen. Bereits im Kindergarten sollen die ersten Maßnahmen gesetzt werden, um die Kleinsten mit einem technischen Verständnis auszustatten. Ein spielerischer Zugang ist der erste Schritt, um die wichtigen Grundkompetenzen zu erlernen und Interesse und Freude an Logik und Technik zu erwecken. Entscheidend ist natürlich die Schullaufbahn. Wir wollen, dass kein Kind die Schule ohne digitale Kompetenzen verlässt. Dafür ist es notwendig, einerseits die technische Infrastruktur zur Verfügung zu stellen, also neben Netzwerk, Servern und Lehrercomputern auch Tablets und Laptops für jedes Kind. Denn es darf nicht von der Dicke der Geldbörse abhängen, ob man die notwendigen Fähigkeiten erlernt oder nicht. Dazu gehört aber auch die Schulung in Medienund Datenkompetenz. Und dafür braucht es nicht zuletzt gut ausgebildetes pädagogisches Personal, denn viel zu oft hört man noch immer, dass sich Lehrer und Lehrerinnen nicht trauen, digitale Lerninhalte zu vermitteln, da sich die Jugendlichen ohnehin weit besser auskennen würden. Daher wird digitale Bildung in Zukunft bei den Ausbildungen deutlich forciert.

Selbstverständlich müssen auch durch Aus- und Weiterbildung im Berufsleben die digitalen Kompetenzen ständig erweitert und ausgebaut werden. Dazu bedarf es auch Umschulungen, die tatsächlich den Anforderungen des Marktes entsprechen. Aber auch Seniorinnen und Senioren sollen den Umgang mit den Neuerungen erlernen. Coachings und Kurse, die mittlerweile in ganz Österreich angeboten werden, helfen dabei.

\section{Arbeitswelt von Digitalisierung durchdrungen}

Die Arbeitswelt ist bereits heute von der Digitalisierung durchdrungen. Bis heute gibt es eine Vielzahl von Studien, die sich mit den Auswirkungen der Digitalisierung auf dem Arbeitsmarkt und die dadurch entstehenden Arbeitsplätze oder den Verlust derselben auseinandersetzen. Je nach Studiendesign, Intention und Auftraggeber kommen die einen Studien zu dem Schluss, dass sich die Anzahl der Arbeitsplätze erhöhen wird, während andere Studien einen massiven Rückgang der Zahl der Arbeitsplätze befürchten. In welche Richtung sich die Veränderung tatsächlich entwickeln wird, ist nicht wirklich vorhersehbar. Die Vergangenheit wurde kürzlich von Agnes Streissler-Führer für das Bundeskanzleramt untersucht. Sie hat sich die Auswirkungen der Digitalisierung auf die Zahl der Arbeitsplätze in den vergangenen 20 Jahren angesehen und ist zu einer interessanten Conclusio gelangt: Grundsätzlich kann nicht von Vorneherein behauptet werden, dass die Digitalisierung mit Arbeitsplatzverlusten einhergeht. Insgesamt sind in den letzten beiden Jahrzehnten sogar mehr Jobs entstanden als im selben Zeitraum verschwunden sind. Ein näherer Blick zeigt, dass in höher digitalisierten Branchen die Zunahme deutlich war, während es in weniger digitalisierten Branchen zu einer Abnahme der Arbeitsplätze kam. Auch wenn man die Zukunft nicht vorhersehen kann, kann man dennoch mit Sicherheit sagen: Die Arbeitswelt wird sich verändern. Und auch hier gilt, gestalten, nicht verwalten. Als Beispielmaßnahmen können wir hier die Förderung von Online-(Berufs-)Weiterbildungskursen zur Vermittlung digitaler Kompetenzen sowie von Umschulungen, die sich an den konkreten Bedürfnissen des Arbeitsmarkts orientieren, nennen. Mit der Wiedereinführung des Fachkräftestipendiums ab 2017, der Ausweitung der arbeitsplatznahen Qualifizierung
(AQUA) sowie der verstärkten Förderung und Unterstützung der betrieblichen Weiterbildung (insbesondere betriebliche Impulsprogramme) werden attraktive Nachqualifizierungsangebote geschaffen. Nicht zuletzt ist der Ausbau von Studienplätzen in MINT-Fächern geplant.

\section{Neue Arbeitsformen}

Und es wird auch notwendig sein, sich den neuen Arbeitsformen zu widmen. Eine Studie der Arbeiterkammer hat gezeigt, dass mittlerweile bereits 18 Prozent der Menschen in Österreich zumindest einmal als Clickworker oder als Crowdworker gearbeitet hat. Firmen wie Uber oder foodora, aber auch Plattformen wie Airbnb sind die bekanntesten Ausformungen dieser neuen Arbeitswelt. Clicken für wenige Cents pro Click, Essenszustellung oder OnlineFahrtendienstvermittlungen sind oftmals für Menschen, die ansonsten keine Arbeit finden oder keine annehmen können, eine gute Möglichkeit für einen Verdienst. Allerdings fehlt hier oftmals jegliche soziale Absicherung. Wir müssen dafür sorgen, dass auch in diesem Bereich das Arbeitsrecht und soziale Standards eingehalten werden. Dies wird allerdings nur auf europäischer Ebene möglich sein, denn diese Arbeitsformen haben sich schon lange von nationalen Grenzen verabschiedet. Daher gilt es, an einer europäischen CrowdworkingRichtlinie zu arbeiten, die die Grundlagen zur sozialen Absicherung von Menschen, die diesen neuen Arbeitsformen nachgehen, festlegt und faire Regeln für Crowdworking definiert.

\section{Innovation als Hebel für den Wirtschaftsstandort}

Gerade für unseren Innovationsstandort ist auch der Start-upBereich wichtig. Hier haben wir sowohl auf Finanzierungs/Investorenseite mit der Mittelstandsfinanzierungsgesellschaft Neu und der Risikokapitalprämie als auch auf Förderungsseite mit 24 h Quickcheck und Extrabudget für akademische Spin-offs Impulse und mit der Förderung der Lohnnebenkosten für die ersten drei Mitarbeiter/innen für Start-ups auch Beschäftigungsanreize gesetzt.

Im Forschungsbereich setzen wir zusätzliche Akzente, wie z. B. mit der Forschungsinitiative Silicon Austria und durch die Entwicklung eines Quantencomputerdemonstrators und vielem mehr.

Wenn wir einen erfolgreichen Wirtschaftsstandort Österreich wollen, bedeutet das für mich nicht Billigproduktion und Lohndumping, sondern Innovation, Forschung, gut ausgebildete Arbeitnehmerinnen, natürlich einen stabilen Rechtsstaat und gut funktionierende Behördenprozesse. Bei all dem kann und soll uns intelligent gestaltete Digitalisierung helfen. Deshalb ist aus meiner Sicht der weitere Ausbau von E-Government so wichtig. Hier sind wir im internationalen Vergleich schon ziemlich gut aufgestellt, aber wir wollen natürlich noch besser werden. Gute, komfortable digitale Services nützen sowohl Bürger/innen als auch Unternehmen; und durch den Einsatz von neuen Technologien kann hier die öffentliche Verwaltung auch Innovationsmotor sein.

Trotz aller Begeisterung für neue Technologien und unseren Anstrengungen, die digitale Kluft zu schließen, ist es entscheidend, dass Behördenwege auch nach wie vor für diejenigen möglich bleiben, die das Internet entweder nicht nützen können oder nicht nützen wollen. Für Bürger/innen haben wir ein Recht auf elektronischen Verkehr mit den Behörden verankert. Ein Recht, aber keine Pflicht, wohl gemerkt. Das wird in manchen anderen Ländern durchaus anders gesehen. Diese Personenanzahl soll jedoch immer geringer werden, damit möglichst viele von den Vorteilen der Digitalisierung etwas haben.

\section{Wir wehren uns gegen Hass im Netz}

Zuletzt sollen aber auch die Schattenseiten des Internets nicht vergessen werden. Wir leben in einer Zeit, in der der Hass wieder stär- 
ker zu werden scheint. Das Internet - und hier speziell die sozialen Medien - haben sich in den vergangenen Jahren teilweise zu regelrechten Hassmaschinen entwickelt. Man hat das Gefühl, im Internet herrscht der "Wilde Westen", mit dem Ergebnis, dass sich die Aggressiven und Lautstarken immer mehr Gehör verschaffen, während sich andere immer weiter zurückziehen. Hier steckt eine potentielle Gefahr für Demokratie und Rechtsstaat. Diesem Phänomen muss man auf mehreren Ebenen begegnen. Zunächst auf einer juristischen. Man darf nicht das Gefühl aufkommen lassen, im Internet handle es sich um einen rechtsfreien Raum, denn das ist nicht so. Selbstverständlich sind Straftaten, die in der realen Welt als solche geahndet werden, auch in der virtuellen Welt strafbar. Dankens- werterweise sind die Bestimmungen in Österreich diesbezüglich gut ausgebaut. Gleichzeitig muss es aber auch zu einer Stärkung der digitalen Zivilcourage kommen. Eintreten für Schwächere und solche, die negative Erfahrungen im Internet gemacht haben, muss zur Selbstverständlichkeit werden. Maßnahmen, wie die im September 2017 eröffnete Beratungsstelle gegen Hass im Netz, liefern hoffentlich einen Beitrag dazu.

Und auch das soll ein Puzzlestein sein, wie die vielen anderen in der Digital Roadmap zusammengefassten, die dazu dienen sollen, dass die Digitalisierung zum Wohle aller und nicht einiger weniger in Zukunft unser Leben prägen wird. 